\title{
Clinical analysis of concurrent 3DCRT and XELOX treatment for postoperative local recurrence of rectal carcinoma in elderly patients
}

\author{
Baoxiong Zhuang ${ }^{1,2}$, Junyao $\mathrm{Xu}^{1,2}$, Kang $\mathrm{Xu}^{1,2}$, Jie Wang ${ }^{1,2}$ \\ 1Department of General Surgery, Sun Yat-sen Memorial Hospital, Sun Yat-sen University, Guangzhou, P. R. China \\ 2Key Laboratory of Malignant Tumor Gene Regulation and Target Therapy of Guangdong Higher Education Institutes, \\ Sun Yat-sen University, Guangzhou, P. R. China
}

Prz Gastroenterol 2013; 8 (4): 257-261 DOI: $10.5114 /$ pg.2013.37489

Key words: rectal carcinoma, recurrence, radiotherapy, pharmacological treatment.

Address for correspondence: Jie Wang, Department of General Surgery, Sun Yat-sen Memorial Hospital, Sun Yat-sen University, No. 107 Yanjiang Western Road, 510120 Guangzhou, China, phone: +86-020-81332199, e-mail: sunswj@yahoo.com.cn

\begin{abstract}
Aim: To probe into the clinical effects of concurrent 3DCRT (3-dimensional conformal radiation therapy) and XELOX treatment for postoperative local recurrence of rectal carcinoma in the elderly patients.

Material and methods: Fifty-seven elderly patients with postoperative local recurrence of rectal carcinoma were randomly divided into a clinical group and a control group. The clinical group had 31 patients, who received $6 \mathrm{MV}$ X-ray 3DCRT, 2 Gy per time, 5 times every week. After radiotherapy for the total pelvis until target dose (DT) in tumor focus reached 40 Gy/20f, the tumor focus was intensified up to DT 60-64 Gy with 8 MV X-ray DT 20-24 Gy. During radiotherapy, the patients were treated with XELOX (oxaliplatin $85 \mathrm{mg} / \mathrm{m}^{2}$, dl, Xeloda $850 \mathrm{mg} / \mathrm{m}^{2}$, p.o. d1-14) for two periods. The control group had 26 patients, who were only treated with XELOX.

Results: The responder rate (RR) of the clinical group was $87.1 \%$. The survival rates within 1,2 and 3 years were $67.7 \%, 51.6 \%$ and $41.9 \%$ respectively, which were higher than the control group $(p<0.05)$. However, the clinical group had significantly more $1^{\text {st }}$ and $2^{\text {nd }}$ degree myelosuppression and hand-foot syndrome. The symptoms were relieved after symptomatic treatment. Conclusions: Concurrent 3DCRT and XELOX treatment for postoperative local recurrence of rectal carcinoma in the elderly patients greatly improved short-term efficacy and survival rate within 1, 2 and 3 years, while the toxic effects also increased greatly, but could be withstood by most patients.
\end{abstract}

\section{Introduction}

The literature indicated that the local recurrence rate of rectal carcinoma after simple radical resection was $4 \%$ to $40 \%$ [1]. The survival period of patients with untreated postoperative local recurrence is generally 3.5 to 13 months [2]. Few patients discover local recurrence early enough to have a second operation. Most patients have not got a second operation opportunity, especially for the elderly patients. Even if local recurrence is found early enough, most patients cannot withstand a second operation due to poor body tolerance [3]. Herein, chemoradiation therapy is especially important for symptom relief, survival time extension and life quality improvement.

\section{Aim}

The aim of the present research was to probe into the clinical effects of concurrent chemoradiation therapy for postoperative local recurrence of rectal carcinoma.

\section{Material and methods}

From Jan 30, 2009 to Sept 30, 2012, our hospital admitted 57 elderly patients with postoperative local recurrence of rectal carcinoma. The 57 elderly patients were randomly divided into a clinical group and a control group. The clinical group had 31 patients, who were treated with concurrent chemoradiation therapy. The control group had 26 patients, who were only treated with XELOX. The 57 patients complied with the follow- 
Table I. Comparison of the clinical and control group patients

\begin{tabular}{|c|c|c|c|}
\hline Patients' features & $\begin{array}{l}\text { Clinical } \\
\text { group } \\
(n=31)\end{array}$ & $\begin{array}{c}\text { Control } \\
\text { group } \\
(n=26)\end{array}$ & $\begin{array}{l}\text { Value of } \\
\qquad p\end{array}$ \\
\hline \multicolumn{3}{|l|}{ Gender } & \multirow[t]{3}{*}{0.940} \\
\hline Male & 14 & 12 & \\
\hline Female & 17 & 14 & \\
\hline \multicolumn{3}{|l|}{ Age } & \multirow[t]{5}{*}{0.421} \\
\hline $60-65$ & 10 & 8 & \\
\hline $66-70$ & 8 & 9 & \\
\hline $71-75$ & 9 & 6 & \\
\hline$\geq 76$ & 4 & 3 & \\
\hline \multicolumn{3}{|l|}{ Pathological class } & \multirow[t]{5}{*}{0.308} \\
\hline Mucinous carcinoma & 2 & 3 & \\
\hline $\begin{array}{l}\text { Middle differentiated } \\
\text { adenocarcinoma }\end{array}$ & 9 & 6 & \\
\hline $\begin{array}{l}\text { Lower differentiated } \\
\text { adenocarcinoma }\end{array}$ & 15 & 8 & \\
\hline $\begin{array}{l}\text { High differentiated } \\
\text { adenocarcinoma }\end{array}$ & 5 & 9 & \\
\hline \multicolumn{3}{|l|}{ Recurrence part } & \multirow[t]{5}{*}{0.683} \\
\hline Anastomoses & 6 & 3 & \\
\hline Perineum tissue & 12 & 8 & \\
\hline Pelvic side lymph code & 8 & 10 & \\
\hline Vagina, uterus, ovary & 5 & 5 & \\
\hline \multicolumn{3}{|l|}{ KPS value } & \multirow[t]{4}{*}{0.126} \\
\hline$\geq 90$ & 9 & 8 & \\
\hline 80 & 14 & 13 & \\
\hline 70 & 8 & 5 & \\
\hline \multicolumn{3}{|l|}{ Original operation mode } & \multirow[t]{3}{*}{0.321} \\
\hline Dixon & 13 & 15 & \\
\hline Miles & 18 & 11 & \\
\hline \multicolumn{3}{|l|}{ Recurrence symptom } & \multirow[t]{5}{*}{0.677} \\
\hline Pain & 6 & 3 & \\
\hline Blood in the stool & 9 & 6 & \\
\hline Weight loss & 11 & 10 & \\
\hline Incomplete obstruction & 5 & 7 & \\
\hline \multicolumn{3}{|c|}{ Recurrence focus size (dia. cm) } & \multirow[t]{4}{*}{0.413} \\
\hline$<2$ & 5 & 10 & \\
\hline $2-4$ & 15 & 17 & \\
\hline$>4$ & 6 & 4 & \\
\hline
\end{tabular}

ing four standards: 1) before the local recurrence, they had radical resection for rectal carcinoma, which could be confirmed with tissue pathology; 2) the local recurrence focus had at least one imaging examination
(Computed tomography - CT), X-ray or endoscopy video) and the focus had a measurable diameter $\geq 10 \mathrm{~mm}$. 3) Before radiotherapy and chemotherapy, cardiograph, blood routine and liver and kidney examination all showed good conditions. 4) Karnofsky (KPS) $\geq 70$; expected survival period $\geq 5$ months. The 2 patient groups are compared in Table I.

A Siemens MD 7745 linear accelerator was used to produce $6 \mathrm{MV}$ X-rays for 3DCRT, 2 Gy per time, 5 times every week. Total pelvis CT scout thin slice contrast enhanced scan was done for ventricumbent patients with full bladder; then the imaging information was input into the 3-dimensional therapy program system (3DTPS). The target area included the rectal tumor, postperitoneal tissue and the $1^{\text {st }}$ and $2^{\text {nd }}$ lymph drainage area at the tumor part. Physicians outlined gross tumor volume (GTV) and clinical target volume (CTV) and important tissues and organs around, including the bladder, pelvic small intestine and thighbone, etc. When the total pelvis achieved DT $40 \mathrm{~Gy} / 20 \mathrm{f}$ with 3DCRT, CT scout contrast enhanced scan was done again with 8MVX 3DCRT, and added DT 20-24 Gy, the tumor DT became 60-64 Gy. The irradiation dosage on small intestine, spinal cord and bladder etc. were within control. Dose volume histogram (DVH) and the equivalent dose curve were used to evaluate the treatment therapy together. The clinical group was treated with XELOX chemotherapy from the first day of radiotherapy. XELOX therapy: oxaliplatin $85 \mathrm{mg} / \mathrm{m}^{2}$, ivdrip day 1 , Xeloda $850 \mathrm{mg} /$ $\mathrm{m}^{2}$, p.o. d1-14 for two periods. The two groups were treated with two periods of chemotherapy when the radiotherapy was over. The chemotherapy: oxaliplatin $130 \mathrm{mg} / \mathrm{m}^{2}$, ivdrip day 1, Xeloda $1250 \mathrm{mg} / \mathrm{m}^{2}$, p.o. d1-14 and one period: 21 days. During the treatment, blood routine was carried out for all patients once every week. Pelvic CT was done when DT was 20 Gy and 40 Gy. Antiinflammation, G-CSF and symptomatic treatment and support treatment etc. were applied to deal with emergency and other complications.

When the treatment was over, CT was done once every two to three months for every patient to observe the tumor volume. The evaluation standard referred to the WHO standard: complete response (CR) was defined as complete disappearance of tumor for more than 4 weeks. Partial response (PR) was defined as more than $50 \%$ regression of tumor volume for more than 4 weeks: for single tumor, two max. vertical lines product; for more than two tumors, the sum of each product. No change (NC) was defined as less than $50 \%$ regression or less than $25 \%$ accretion of tumor volume. Progressive disease (PD) was defined as more than 25\% accretion of tumor volume or appearance of a new focus. $R R$ (objective responder rate) $=C R+P R$. Side ef- 
fects were classed according to the Radiation Therapy Oncology Group (RTOG) toxic standard (CTC2.0 version).

\section{Statistical analysis}

SPSS 13.0 statistics software was used for data processing. The $\chi^{2}$ test was used for comparison of categorical variables between groups. Survival analysis used the Kaplan-Meier method. It was regarded to have statistical significance when $p<0.05$.

\section{Results}

\section{Follow-up}

The follow-up started from the date when patients were admitted to our hospital and ended with patients' death or on Sept 30, 2012. Follow-up rate: 100\%. Median follow-up period was 28 months (3 to 44 months).

\section{Short-term efficacy}

All patients in the clinical group and the control group finished radiotherapy and chemotherapy as regulated. Within 2 months after treatment, the clinical group had 11 CR (35.5\%) and 16 PR (51.6\%) and RR was $87.1 \%$, significantly higher than the control group: RR $65.4 \%$ $(p<0.05)$. The relief rate of pain, blood in the stool and incomplete obstruction in the clinical group and the control group were $100 \%$ and $100 \%, 88.9 \%$ and $83.3 \%$, 80\% and $85.7 \%$. The symptom relief between the two groups had no significant difference $(p>0.05)$ (Table II).

\section{Long-term efficacy}

Up to the end of follow-up on Sept 30, 2012, the clinical group had 18 deaths and 13 survivals and the control group had 21 deaths and 5 survivals. The survival rate of the clinical and control groups within 1, 2 and 3 years were $67.7 \%$ and $42.3 \%, 51.6 \%$ and $30.8 \%, 41.9 \%$ and $19.2 \%$, respectively. The survival rate of the clinical group and control group had a significant difference $(p<0.05)$ (Table III).

\section{Toxic effects}

Main toxic effects included $1^{\text {st }}$ and $2^{\text {nd }}$ degree myelosuppression, hand-foot syndrome and radiation enterocolitis. Third degree toxic and side effects were not available. The clinical group had significantly more myelosuppression and hand-foot syndrome than the control
Table II. Short-term efficacies of the clinical and control groups

\begin{tabular}{lcc}
$\begin{array}{l}\text { Variable } \\
\text { Suppression of tumor }\end{array}$ & $\begin{array}{c}\text { Clinical } \\
\text { group }\end{array}$ & $\begin{array}{c}\text { Control } \\
\text { group }\end{array}$ \\
\hline CR & $11(35.5 \%)$ & $8(30.8 \%)$ \\
\hline PR & $16(51.6 \%)$ & $9(34.6 \%)$ \\
\hline NC & $2(6.5 \%)$ & $6(23.1 \%)$ \\
\hline PD & $2(6.5 \%)$ & $3(11.5 \%)$ \\
\hline RR & $27(87.1 \%)$ & $17(65.4 \%)$ \\
\hline Symptom relief rate & $3 / 3(100 \%)$ & $3 / 3(100 \%)$ \\
\hline Blood in the stool & $8 / 9(88.9 \%)$ & $5 / 6(83.3 \%)$ \\
\hline Incomplete obstruction & $4 / 5(80 \%)$ & $6 / 7(85.7 \%)$
\end{tabular}

group $(p<0.05)$. No patients in the two groups had a hepatotoxic, nephrotoxic or allergic response. There was no treatment-related death (Table IV).

\section{Discussion}

Currently surgical operation is the best choice for rectal carcinoma patients, although the survival rate within 5 years is only about $50 \%$, mostly due to local recurrence. Local recurrence generally emerges earlier than distant recurrence. Sixty-eighty percent of local recurrence occurs within 2 years after the operation. Most recurrence occurs within 8 to 22 months after the operation [4]. Some recur within intestinal anastomoses; and most recur outside the intestine and invade anastomoses and even pelvic soft tissue and surrounding organs and tissues. Recurrent tumor invasion or nerve compression causes pain, obstruction, frequent stool, blood in stool, etc., and severely influences patients' life quality and survival time. The natural survival time of local recurrence of rectal carcinoma patients is generally 7 months. In the literature [5] it is reported that some advanced rectal cancer patients or recurrence patients had a relatively long survival time after overall pelvic organs dissection. However, the great surgical trauma makes it hard for advanced rectal cancer patients to accept surgical treatment. In recent years, with the emergence of new anti-cancer drugs and development of radiotherapy technology, treatment effects of rectal carcinoma have improved greatly.

Table III. Survival rate of the clinical and control groups

\begin{tabular}{lcccc} 
Group & No. & Survival rate within 1 year & Survival rate within 2 years & Survival rate within 3 years \\
\hline Clinical & 31 & $21(67.7 \%)$ & $16(51.6 \%)$ & $13(41.9 \%)$ \\
\hline Control & 26 & $11(42.3 \%)$ & $8(30.8 \%)$ & $5(19.2 \%)$ \\
\hline Value of $p$ & & $<0.05$ & $<0.05$ & $<0.05$
\end{tabular}


Table IV. Toxic effects of the clinical and control groups

\begin{tabular}{lccc} 
Toxic effects & $\begin{array}{c}\text { Clinical } \\
\text { group }\end{array}$ & $\begin{array}{c}\text { Control } \\
\text { group }\end{array}$ & $\begin{array}{c}\text { Value of } \\
p\end{array}$ \\
\hline Myelosuppression & $27(87.1 \%)$ & $7(26.9 \%)$ & $<0.05$ \\
\hline Hand-foot syndrome & $18(58.1 \%)$ & 0 & $<0.05$
\end{tabular}

Nowadays, 3-dimensional conformal radiation therapy (3DCRT) is widely used. While the target tumor area receives a large dose of radiation (up to $60 \mathrm{~Gy}$ ), the surrounding normal tissues can be protected to a large extent and complications can be reduced. However, radiotherapy is only a local therapy and cannot relieve distant metastases. At present, clinical examination cannot detect many micro-metastases, which might influence patients' long-term survival [6]. Now it has become a research focus of concurrent chemoradiation therapy for postoperative local recurrence of rectal carcinoma.

Concurrent chemoradiation therapy can not only relieve local symptoms but also control general disease development. The NSABPR-2 study [7] indicated that concurrent chemoradiation therapy can improve the survival rate or local control rate. The mechanism may be as follows [8]: chemotherapy acts on various phases of tumor cells, disturbs tumor cells' proliferation dynamics and controls cells' rate of proliferation after radiotherapy; therefore, tumor cells enter into a radiation sensitive cycle from the insensitive period and the sublethal damage repair and potential lethal damage repair are controlled. Tumor volume decreases and cell oxygen supply improves and increases hypoxic cells' sensitivity. Thereby, concurrent chemoradiation therapy improves the local control rate and reduces recurrence and metastasis and ameliorates short-term efficacy and long-term survival rate. Toxic and side effects also increase but can be withstood by most patients.

Common concurrent chemoradiation therapy for advanced and postoperative local recurrence of rectal carcinoma is CF + 5-FU; however, this therapy requires long and continuous drip intravenous, which brings much trouble to patients and has strong side effects. Patients often fail to follow up this therapy [9]. Xeloda is an oral fluoropyrimidine drug and has no cytotoxicity; while with TP, Xeloda turns into 5-Fu [10] in the tumor, which can cut into the tumor DNA strand and disturb its replication and repair and provide highly targeted tumor treatment. Radiation therapy can upgrade tumor TP activity, while it does not upgrade normal tissue TP activity; thus, TP content and activity of tumor is higher than normal tissues. Xeloda has better targeting ability than 5-FU; therefore, concurrent radiotherapy and Xeloda can promote sensitization and treat tumor. Mean- while, Xeloda can prevent tumor metastasis during radiotherapy. Multiple clinical studies [11-13] have proved the safety and reliability of concurrent Xeloda and radiotherapy. Oxaliplatin is a $3^{\text {rd }}$ generation platinum complex. In vitro and in vivo clinical studies indicate that oxaliplatin can significantly suppress a large intestine cancer cell line. Xeloda and oxaliplatin have strong synergistic effects on colorectal cancer. Sawada found in in vitro research that XELOX (Xeloda and oxaliplatin) can suppress CXF280 large intestine cancer cells better than an individual drug. Oxaliplatin can upgrade CXF280 large intestine tumor cell TP, which may speed up conversion of Xeloda to 5-FU. Xeloda and oxaliplatin have a synergistic effect $[14,15]$. Rodel et al. conducted research on use of concurrent radiotherapy and XELOX to treat 32 patients with local advanced large intestine cancer. The results indicated that in $55 \%$ of patients TNM decreased progressively and they could undergo a surgical operation. The postoperative sample indicated that $19 \%$ of patients showed a pathological complete response (CR) and $79 \%$ of T4 patients could undergo radical surgery. Therefore, Rodel et al. deemed that concurrent XELOX and 3DCRT was safe and can be standard therapy for fluorouracil-based chemoradiation therapy.

The present research used concurrent 3DCRT and XELOX therapy and the results indicated that this therapy's efficacy is $87.1 \%$. Survival rates within 1,2 and 3 years were $67.7 \%, 51.6 \%$ and $41.9 \%$, significantly higher than the control group $(p<0.05)$. First and second degree myelosuppression and hand-foot syndrome in the clinical group were significantly more frequent than in the control group. The symptoms were relieved after symptomatic treatment. Other severe toxic effects were not available.

The present research indicated that concurrent 3DCRT and XELOX therapy can effectively improve rectal carcinoma postoperative local recurrence rate (RR) and survival rate. Due to the toxic effects, individualized therapy should be made according to patients' integral conditions, such as body constitution and cardiopulmonary function, to improve patients' life quality, relieve pain and extend survival time.

\section{Acknowledgments}

Grant support: Scientific and technological projects in Guangdong (NO. 2008A03021005).

\section{References}

1. Radice E, Dozois RR. Locolly recurrent recial cancer. Dig Surg 2001; 18: 355-62.

2. Saito N, Koda K, Takinguchi N, et al. Curative surgery for local pelvic recurrence of rectal cancer. Dig Surg 2003; 20: 192-200.

3. Gollins S. Radiation, chemotherapy and biological therapy in the curative treatment of locally advanced rectal cancer. Colorectal Dis 2010; 12 Suppl 2: 2-24. 
4. Bisceglia G, Mastrodonato N, Rucci B, et al. Effectiveness of neoadjuvant radiotherapy in the treatment of locally advanced rectal cancer: a single-center experience in 263 patients. Dig Surg 2010; 27: 217-23.

5. Liu YL, Yang YM, Wang XS. Treatment strategy and pattern evaluation of 314 patients with recurrence of rectal cancer. Zhonghua Wei Chang Wai Ke Za Zhi 2010; 13: 572-6.

6. Huerta S. Rectal cancer and importance of chemoradiation in the treatment. Adv Exp Med Biol 2010; 685: 124-33.

7. Wolmark N, Wieand HS, Hyams DM, et al. Randomized trial of postoperative adjuvant chemotherapy with or without radiotherapy for carcinoma of the rectum: National Surgical Adjuvant Breast and Bowel Project Protocol R-02. J Natl Cancer Inst 2000; 92: 388-96.

8. Lee SJ, Ahn BM, Kim JG, et al. Definitive chemoradiotherapy with capecitabine and cisplatin in patients with esophageal cancer: a pilot study. J Korean Med Sci 2009; 24: 120-5.

9. Ramani VS, Sun Myint A, Montazeri A, et al. Preoperative chemoradiotherapy for rectal cancer: a comparison between intravenous 5-fluorouracil and oral capecitabine. Colorectal Dis 2010 Aug; 12 Suppl 2: 37-46.

10. Ebi H, Sigeoka Y, Saeki T, et al. Pharmacokinetic and pharmacodynamic comparison of fluoropyrimidine derivatives, capecitabine and 5'-deoxy-5-fluorouridine(5'-DFUR). Cancer Chemother Pharmacol 2005; 56: 205-11.

11. Sauer R, Becker H, Hohenberger W, et al. Preoperative versus postoperative chemoradiotherapy for rectal cancer. N Engl I Med 2004; 351: 1731-40.

12. Souglakos J, Androulakis N, Mavroudis D, et al. Multicenter dose-findling study of concurrent capecitabine and radiotherapy as adjuvant treatment for operable rectal cancer. J Int J Radiat Oncol Biol Phys 2003; 56: 1284-7.

13. Dunst J, Debus J, Rudat V, et al. Neoadjuvant capecitabine combined with standard radiotherapy in patients with locally advanced rectal cancer: mature results of a phase II trial. Strahlenther Onkol 2008; 184: 450-6.

14. Gassidy J, Tabernero J. XELOX(capecitabine plus oxaliplatin): active first-line therapy for patients with metastatic colorectal cancer. J Clin Oncol 2004; 22: 2084-91.

15. Rodel C, Grabenbauer GG, Papadopoulos T, et al. Phase I/II trial of capecitabine, oxaliplatin, and radiation for rectal cancer. J Clin Oncol 2003; 21: 3098-104. 